\section{ORIGINAL RESEARCH}

S.S. Yom

A.S. Garden

G.A. Staerkel

L.E. Ginsberg

W.H. Morrison

E.M. Sturgis

D.I. Rosenthal

J.N. Myers

B.S. Edeiken-Monroe

\title{
Sonographic Examination of the Neck after Definitive Radiotherapy for Node-Positive Oropharyngeal Cancer
}

BACKGROUND AND PURPOSE: Radiographic determination of viable disease in cervical adenopathy following RT for head and neck cancer can be challenging. The purpose of this study was to evaluate the utility of US, with or without FNA, in regard to the postradiotherapy effects on documented metastatic adenopathy in patients with oropharyngeal cancer.

\begin{abstract}
MATERIALS AND METHODS: This study included 133 patients with node-positive oropharyngeal cancer who were irradiated from 1998 to 2004. Sonographic evaluation was performed within 6 months of completion of radiation. Posttreatment US results were compared with pretreatment CT images and were recorded as the following: progression, suspicious, indeterminate, posttreatment change, or regression (positive) versus nonsuspicious or benign (negative). FNAC was classified as nondiagnostic, negative, indeterminate, or positive. Results of US and US-guided FNAC were correlated with findings at neck dissection and disease outcome.
\end{abstract}

RESULTS: Of 203 sonographic examinations, 90\% were technically feasible and yielded a nonequivocal imaging diagnosis. Of 87 US-guided FNAs, 71\% yielded a nonequivocal tissue diagnosis. The PPV and NPV of initial posttreatment US were $11 \%$ and $97 \%$. Sensitivity and specificity were $92 \%$ and $28 \%$. The PPV and NPV of US-guided FNA were $33 \%$ and $95 \%$, and the sensitivity and specificity were $75 \%$ and $74 \%$. On serial sonographic surveillance, of 33 patients with nonsuspicious findings, only 1 (3\%) had neck recurrence. Of 22 patients with questionable findings on CT and negative findings on US, none had a neck recurrence.

CONCLUSIONS: In experienced hands, serial US is an inexpensive noninvasive reassuring follow-up strategy after definitive head and neck RT, even when CT findings are equivocal.

\begin{abstract}
ABBREVIATIONS: FNA $=$ fine-needle aspiration; FNAC = fine-needle aspiration cytology; late recur $=$ late recurrence, $>6$ months later; NOS = not otherwise specified; NPV = negative predictive value; MDACC $=$ M.D. Anderson Cancer Center; PET = positron-emission tomography; $\mathrm{PPV}=$ positive predictive value; $\mathrm{RT}=$ radiation therapy; $\mathrm{US}=$ ultrasonography
\end{abstract}

$\mathbf{U}_{\mathrm{s}}^{\mathrm{s}}$ examination has been found to be a valuable technique in the staging of patients with head and neck cancer. ${ }^{1-3}$ Three comparative studies showed that US produced a higher sensitivity and comparable specificity in the initial distinction of metastatic adenopathy from head and neck cancer than either clinical examination or diagnostic CT of the head and neck. ${ }^{4-6}$ The argument has also been presented for the use of US as an initial screening examination before CT for the staging of oral cavity cancer. ${ }^{7}$ When combined with US for initial staging, US-guided FNAC has a high overall diagnostic accuracy of $95 \%$ for head and neck adenopathy. ${ }^{8}$

Management of the neck for patients with carcinoma of the oropharynx with cervical metastases is evolving. Historically, most patients would be irradiated and then undergo a planned neck dissection. More recently, many clinicians are advocating

Received June 2, 2010; accepted after revision December 29.

From the Departments of Radiation Oncology (A.S.G., W.H.M., D.I.R.), Diagnostic Radiology (L.E.G., B.S.E.-M.), Pathology (G.A.S.), Head and Neck Surgery (E.M.S., J.N.M.), and Epidemiology (E.M.S.), The University of Texas M.D. Anderson Cancer Center, Houston, Texas; and the Department of Radiation Oncology (S.S.Y.), University of California, San Francisco, San Francisco, California.

Paper previously presented in part at: Annual Meeting of the American Radium Society, May 3-7, 2008; Dana Point, California.

Please address correspondence to Adam Garden, MD, Division of Radiation Oncology, Box 97, The University of Texas M.D. Anderson Cancer Center, 1515 Holcombe Blvd, Houston, TX 77030; e-mail: agarden@mdanderson.org

http://dx.doi.org/10.3174/ajnr.A2545 basing the decision to perform a neck dissection on the response to therapy. For patients with a complete response, observation is recommended, with neck dissection reserved for those with residual adenopathy. Most clinicians have relied on CT or MR imaging to make this decision. However, even in patients with less than a complete response, pathologic evaluation of the neck-dissection specimen often reveals only fibrosis and/or nonviable tumor. Postradiation, many patients present with CT scans in which there is a small nodal remnant of uncertain viability. To better predict which patients would require a neck dissection, we often required that patients undergo complementary radiographic examinations that might give us additional information regarding the status of the nodes in the neck.

US of the neck was often chosen in these situations. It was attractive because with experienced sonographers, it provided a view of the soft tissues of the neck and is noninvasive. US examination of the neck is not, however, an established means of assessment after a course of definitive RT. Scientific literature on this subject is scant, and for most clinicians in the United States, US is not a tool that inspires great confidence in the postradiotherapy setting.

Nonetheless, US in combination with US-guided FNAC has been used in combination with CT at the University of Texas MDACC for many years as a supplementary means of assessing the irradiated neck. The purpose of this study was to 


\begin{tabular}{lc}
\hline Table 1: Patient and tumor characteristics & \\
\hline Characteristic & No. \\
\hline Sex & 112 \\
Male & 21 \\
Female & 54 (30-82) \\
Age (median) (range) (yr) & \\
Primary Site & 56 \\
Tonsil & 67 \\
Base of tongue & 3 \\
Pharyngeal wall & 4 \\
Soft palate & 3 \\
Oropharynx NOS & \\
Clinical T Stage & 1 \\
CTis & 36 \\
CT1 & 47 \\
CT2 & 24 \\
CT3 & 16 \\
CT4 & 9 \\
NOS & \\
Clinical N Stage & 16 \\
N1 & 23 \\
N2a & 52 \\
N2b & 22 \\
N2C & 17 \\
N3 & 3 \\
NOS & \\
\hline
\end{tabular}

\begin{tabular}{lc}
\hline \multicolumn{2}{l}{ Table 2: Treatment and follow-up characteristics } \\
\hline Characteristic & No. \\
\hline Technique & 72 \\
3D conformal & 54 \\
Intensity-modulated & 3 \\
Ipsilateral only & 4 \\
$\quad$ NOS & $70(64-75.6)$ \\
Dose to primary site (Gy) (median) (range) & $41(35-61)$ \\
Radiation treatment time (median) (range) (days) & $61(26-187)$ \\
Days to first US (median) (range) & $53(9-101)$ \\
Postradiotherapy follow-up (median) (range) (mo)
\end{tabular}

determine the degree and quality of the diagnostic contributions of US and US-guided FNAC specifically for node-positive oropharyngeal cancers treated with definitive RT.

\section{Materials and Methods}

\section{Patients}

Medical records were retrospectively reviewed for 1279 patients with oropharyngeal cancer who were treated with definitive RT at MDACC from 1998 to 2004. Among the 1042 patients who had nodal disease at presentation, 679 had no surgery on their necks before radiation or chemoradiation. Of these patients, 133 had at least 1 follow-up US within 6 months of completion of radiation treatment. Patients were predominantly male with base of tongue and tonsillar tumors, and most had N2-N3 nodal disease (Table 1). While the treatment technique was either 3D-conformal or intensity-modulated RT, parameters were fairly consistent (Table 2). An institutional waiver of informed consent was obtained for review of patient records within this cohort, and the institutional review board approved the study.

\section{Pretreatment Evaluation}

All patients had an initial staging work-up consisting of history and physical examination, laboratory studies, CT and/or MR imaging of the head and neck, chest radiography or CT, pretreatment dental evaluation, and biopsies and pathologic review for diagnosis. Sixteen patients had pretreatment US to assist in staging of the neck.

\section{Follow-Up}

Patients were seen at approximately 6 weeks following completion of their radiation for their initial restaging. Patients, as described above, are staged on presentation with CT, and patients postradiation are rescanned to assess treatment response. During the years of this study, US was used in selected patients to further evaluate the neck. Two hundred seventy patients had a complete response on CT. Twentytwo $(8 \%)$ of these patients underwent US to provide additional confidence in the CT interpretation of response. The remaining patients in our cohort had US examinations to assess residual adenopathy to better predict whether the nodal architecture appeared benign or malignant or to use US as a guide for localization of the node for USguided aspiration purposes. The guidelines for the request of US were based on the treating clinicians' judgment of a patient's response and/or CT findings of concern for persistent adenopathy. Infrequently, US was used when clinical or radiographic examination demonstrated either new adenopathy or new progression in a lymph node that had initially demonstrated a response.

The median time to US examination after RT completion was 61 days (range, 26-187 days). The median posttreatment follow-up duration was 53 months (range, 9-101 months) (Table 2).

Postradiation US examination was conducted by experienced and specialized members of the diagnostic imaging faculty at MDACC. US examination of the soft tissues of the neck was performed by using a high-frequency linear-array transducer of at least 7 and $\leq 13 \mathrm{MHz}$, connected to a high-resolution scanner (Alpha 10; Aloka, Tokyo, Japan). US examination included a focused evaluation of the region of the lymph nodes in the bilateral jugular territories, superior neck, and parotid glands.

A combination of multidirectional and color duplex US techniques, including gray-scale, color Doppler, and power Doppler imaging, was used as indicated. ${ }^{9-11}$ Pathologic findings were captured with freeze-frame imaging on screens for later review (Figs 1 and 2). Criteria used to guide nodal evaluation were based on the size; shape; echogenicity; an absent, distorted, or displaced central hilum; and/or disorganized intranodal vascularity (Table 3 ). ${ }^{12,13}$

The size of examined lymph nodes ranged from 0.6 to $4 \mathrm{~cm}$. On the initial, second, third, and fourth sequential posttreatment US examinations, at 6-month intervals, the median size of the largest node measured 2.0, 1.6, 1.7, and $0.8 \mathrm{~cm}$, respectively.

An US-guided FNA was performed when there was concern for residual adenopathy based on the US criteria of a metastatic lymph node. FNA was performed by the operator on the basis of the imaging findings and the clinical judgment of the multidisciplinary oncology team.

FNA was not always performed after suspicious US findings if it was judged unnecessary on the basis of compelling US characteristics. In these cases, FNA was not performed because the surgeon would perform neck dissection independent of the FNA result. Alternatively, if the US examination revealed a benign lymph node, an FNA was often not performed because there was no target identified by US to biopsy.

Following the instillation of approximately $2 \mathrm{~mL}$ of local anesthetic agent, aspiration was performed with an 18-ga needle attached to a 20 -mL syringe. A single needle insertion was made for each patient. The needle tip was placed within the node of concern, after which aspiration was performed. Aspiration consisted of 

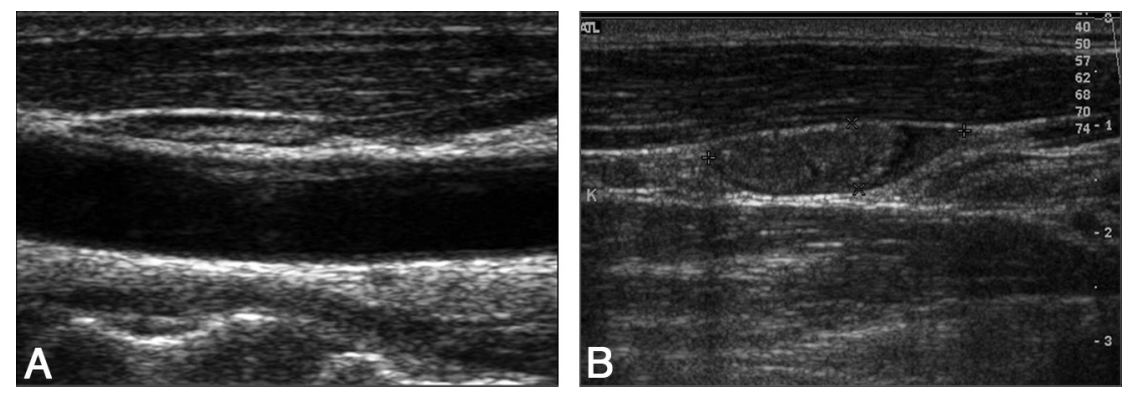

Fig 1. A, Normal lymph node, with a flat echogenic hilum. $B$, Metastatic lymph node, with plump gray infiltration of the hilum.
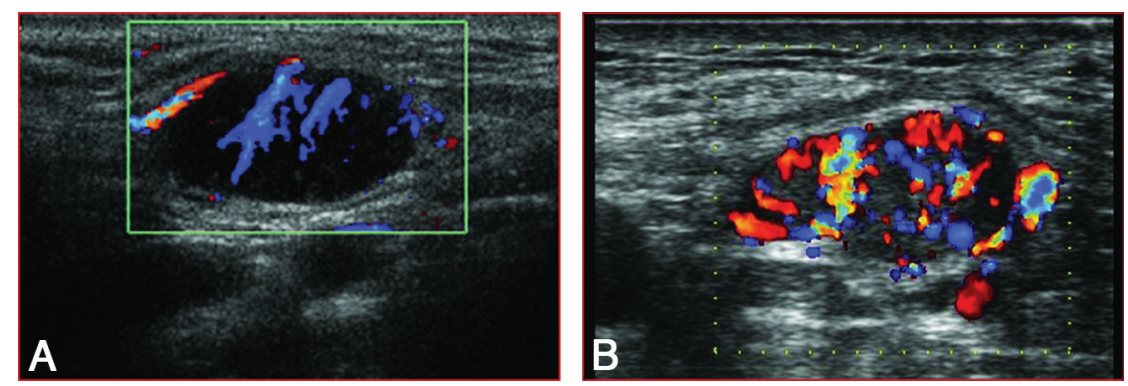

Fig 2. A, Normal lymph node, with organized vascular flow. B, Metastatic lymph node, with disorganized subcapsular flow.

\begin{tabular}{ll}
\hline $\begin{array}{l}\text { Table 3: Criteria for malignancy in US evaluation of the } \\
\text { postradiotherapy neck }\end{array}$ \\
\hline Characteristic \\
\hline Size & $>7-8 \mathrm{~mm}$ \\
Shape & Rounded, long-/short-axis ratio $<2.0$ \\
Hilum & Nonechogenic, infiltrated \\
Outline & Presence of extracapsular extension \\
Necrosis & Cystic or focal defects \\
Color flow & Disorganized, subcapsular flow \\
History & Change from prior exam \\
\hline
\end{tabular}

back-and-forth movements of the needle while maintaining its location within the node and simultaneously applying suction by pulling on the plunger of the attached syringe. Aspirated material was placed onto glass slides and fixed in modified Carnoy solution. Slides were then stained with the Papanicoloau method. An immediate assessment for specimen adequacy and preliminary diagnosis was made by an attending cytopathologist who, in turn, informed the radiologist of the results. Insufficient specimens underwent a second aspiration.

\section{Scoring and Statistical Methods}

Results of US studies were abstracted from impressions recorded at the time of the examination. These were classified as the following: progression, suspicious, indeterminate, posttreatment change, or regression (group 1, positive) versus nonsuspicious or benign (group 2, negative). FNAC results were coded as nondiagnostic, negative, indeterminate, or positive. Coterminous clinical impressions, CT findings, pathologic findings of subsequent neck dissection, and disease outcome were also recorded and compared with the US interpretation and result of the FNA of the posttreatment targeted lymph node.

Descriptive statistics were performed by using the Statistical Package for the Social Sciences, Version 16.0 (SPSS, Chicago, Illinois).

\section{Results}

\section{Feasibility of US and FNA in the Irradiated Neck}

A total of 203 neck US examinations were performed. One hundred thirty-three patients had initial US at 3- to 6-month intervals; 52 patients underwent a second US; and 14, a third US. One patient had a fourth and fifth US, and 1 patient had 2 US examinations after neck dissection. Only 1 examination $(0.5 \%)$ could not be completed due to technical inability to assess the location of interest. Twenty examinations yielded visual results that were deemed indeterminate by the operator $(10 \%)$, while the remaining examinations resulted in a more precise level of diagnostic certainty.

A total of 87 postradiotherapy FNA procedures were performed. Among these attempts, 2 failed to obtain a specimen, 16 were nondiagnostic, and 7 were deemed indeterminate (including the inability to exclude metastasis or finding atypical cells that could otherwise not be qualified). The remaining 62 (71\%) procedures yielded a positive or negative diagnosis.

\section{Predictive Value of Postradiation US}

Of 132 patients who underwent and completed an initial postradiation US, 97 had examination findings that were considered positive (Fig 3). Thirty-two (33\%) underwent neck dissections, and 9 had specimens positive for recurrence. Two of the remaining 65 patients who did not undergo immediate neck dissections subsequently had disease recur in their necks.

Thirty-five patients had an initial US interpreted as nonsuspicious. One patient had an immediate neck dissection, the findings of which were negative. Another patient had initial US with negative findings but, several months later, due to continuing clinical suspicion, underwent a neck dissection that was positive for recurrence.

Fifty-two patients continued to have subsequent follow-up US (0.6-81.2 months following their initial US) for a total of 67 examinations. Thirty-four findings were positive, and 6 


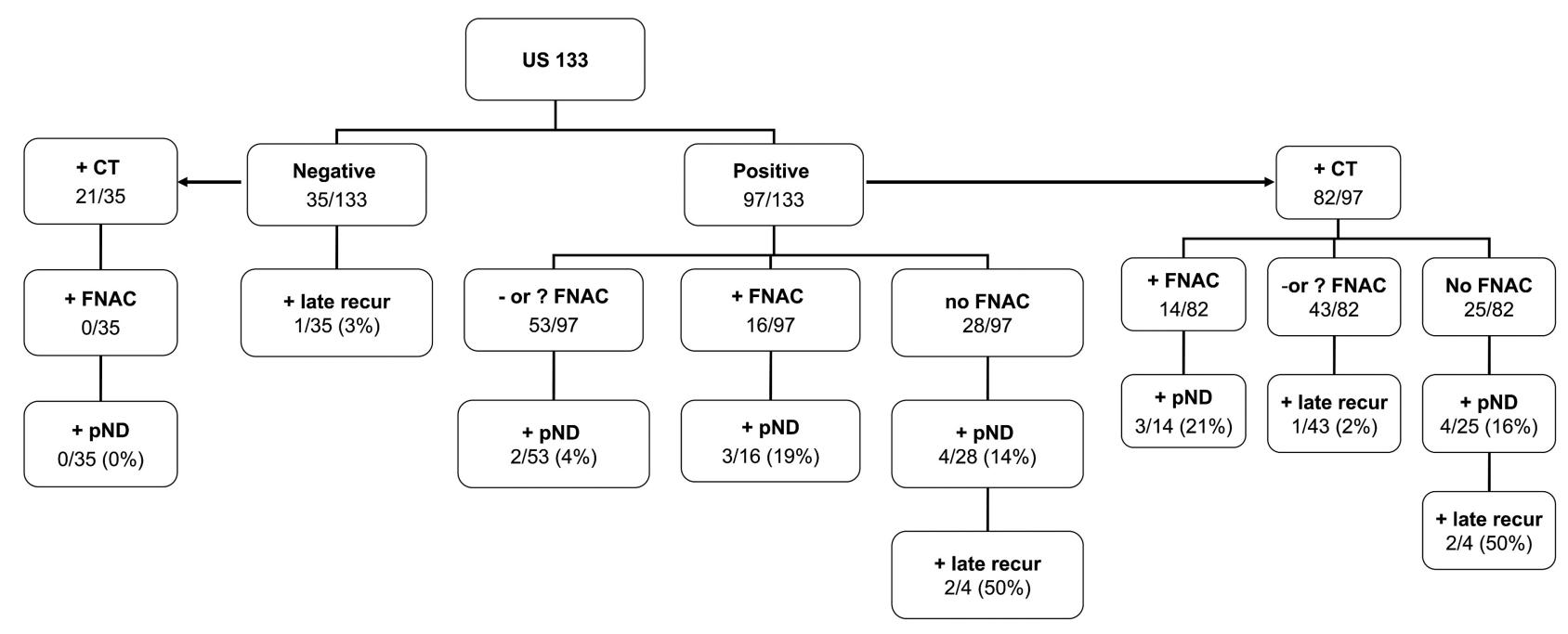

Fig 3. Flow chart of patient evaluation results..

patients went on to have either neck recurrence or positive disease at neck dissection. Findings of 33 tests were negative, and only 1 patient from this group (who had discordant results on serial examination) went on to develop a neck recurrence.

The PPVs and NPVs of initial posttreatment US in our patients were $11 \%$ and $97 \%$, respectively. The sensitivity and specificity were $92 \%$ and $28 \%$, respectively.

\section{Predictive Value of US Combined with FNAC}

Among the 131 US examinations with positive findings, FNAC studies followed in 79. Findings of 19 FNAC specimens $(25 \%)$ in 18 patients were read as unequivocally positive. Seventeen patients had neck dissections, of which 5 had specimens positive for recurrence. One patient eventually had clinical evidence of disease in the neck but also had primary site disease, so salvage neck dissection was not performed.

FNAC was interpreted as nondiagnostic or indeterminate in 21 patients and failed to obtain specimens in 2 patients. Among these 23 patients, 7 had neck dissections, all of which had negative findings, and none of these patients had a recurrence in their necks.

FNAC findings were read as unequivocally negative in 37 patients. No patient in this group underwent immediate dissection, but on subsequent evaluations, 4 patients had clinical findings and/or imaging that was suspicious for disease and eventually underwent neck dissections. Two patients had negative findings on dissections, while 2 had all negative nodes but cancer was identified within muscle, 1 of these in a distant area than had been examined on US.

PPV and NPV values of FNA cytopathology in our patients with positive findings on US were $33 \%$ and $95 \%$, respectively. The sensitivity and specificity were $75 \%$ and $74 \%$, respectively.

In the 35 patients with initial US findings considered negative, 3 patients (9\%) underwent aspiration. Findings of 2 of these were negative, and 1 aspiration fortuitously found a new thyroid cancer in the thyroid gland. Among the 29 patients with 33 follow-up US findings considered negative, 2 patients underwent FNAC. Findings of 1 were considered suspicious, and that patient went on to develop neck recurrence.

\section{Comparison of CT and US Findings}

One hundred twenty patients had a CT scan at the time of the first US. The single patient who could not be assessed by US due to inaccessibility of the location of interest did undergo a CT scan, the findings of which were considered positive.

Eighty-two patients had a CT demonstrating residual adenopathy at the time of positive US findings, and 57 of them underwent FNAC. Fourteen had positive findings on cytopathology, of whom 13 underwent neck dissections and 3 had neck disease at neck dissection. Therefore, 3 of 14 (21\%) patients with positive findings on CT, US, and FNAC had positive findings on neck dissections. Four patients had an indeterminate cytopathology, and 3 underwent neck dissections; findings of all 3 dissections were negative, and all 4 patients were controlled in the neck. In 12 patients, there was failure to obtain a specimen or the specimen was nondiagnostic, resulting in 3 negative findings on neck dissections; all 12 patients had disease controlled in the neck. Twenty-seven patients had negative findings on FNAC, resulting in 2 negative findings on neck dissections and 1 patient who had subsequent CT-identified neck recurrence 15 months later in a different area. Therefore, of the 43 patients with positive findings on CT and US but nonpositive or equivocal FNAC findings, 1 (2\%) patient had disease recur in the neck.

Of the 25 patients with positive findings on US and CT who did not have FNA, 5 went to immediate neck dissections due to a high level of clinical suspicion, of which 4 were positive for recurrence. Another patient underwent subsequent US with findings deemed suspicious and FNAC confirming neck recurrence, and another refused further work-up or surgery but experienced recurrence in the primary site and then the neck.

Ten patients had negative initial findings on US and CT; 1 of these patients had subsequent positive findings on US, resulting in positive findings on neck dissection.

Twenty-eight patients had discordance between their initial US and CT. Five had negative findings on CT but suspicious findings on US; all remained free of neck disease. Another patient had suspicious findings on CT with an inability to assess the neck by US; this patient experienced recurrence in 
the neck. Two patients had suspicious CT findings, but their US findings were benign; neither experienced neck recurrence. Twenty patients had indeterminate, posttreatment, or regressing nodes on CT but entirely negative findings on US, among which there were 3 negative findings on FNAC and 1 cytopathology read as atypical. One of these patients had negative findings on neck dissection, and no patient had a recurrence in the neck. Therefore, even in the face of suspicious or questionable CT findings, there was a very high NPV (100\%) of benign findings on US.

\section{Discussion}

Management of the neck after RT continues to be a controversial issue, plagued by the dilemmas of postradiation diagnostic uncertainty versus the appeal of prompt neck dissection to address a potential regional failure. Historically, some institutions have advocated a planned neck dissection for all patients with evidence of stage $\mathrm{N} 2$ or N3 adenopathy at presentation $^{14-16}$ due to improved regional disease control rates seen with the addition of neck dissection after RT. ${ }^{17,18}$ We and others have, in the absence of clinically or radiographically evident disease, advocated close monitoring of the neck, even for those initially staged as $\mathrm{N} 2$ or N3. ${ }^{19-22}$

The criteria for proceeding with neck dissection in the case of a partial clinical or radiographic response are not well-established. CT and MR imaging often yield equivocal findings based on size criteria, though some standards for radiographic evaluation of the neck are starting to emerge. ${ }^{23}$ PET has recently been advocated as the technique of choice to determine if neck dissection is necessary postradiation, but this technique has not yet been validated. PET is hampered by the high rates of false positivity in the first few months after RT and the concern for false negativity in very small tumor residuals. A recent prospective study from our institution suggested that PET-CT did not offer a significant advantage over contrastenhanced CT in determining the need for neck dissection postradiation. ${ }^{24}$

Sonography with or without FNAC is an underappreciated means of imaging for the presence of neck nodal disease. US examination of the neck offers some obvious practical advantages, including a lack of ionizing radiation exposure, relative portability, low cost in comparison with other types of imaging, the ability to adapt dynamically to a hands-on exploration of unusual or unexpected patient anatomy or technically accessible suspicious findings, and the ability to get an immediate answer. ${ }^{25}$ Some have argued that US can be more effectively performed by clinicians who have the advantage of correlating the imaging with a patient's known history. ${ }^{26} \mathrm{Fi}$ nally, US has the unique advantage of real-time pathologic correlation via US-guided FNAC, which can be instantly analyzed and, if necessary, repeated and confirmed with multiple passes within the timeframe of a single study. ${ }^{27}$

For reasons of cost and efficiency, US generally has been used more liberally in Europe than in the United States ${ }^{28,29}$ and has been proposed as a follow-up strategy for the neck in some international forums. ${ }^{30,31}$ Specifically in the postradiation setting, one older study established that the effects of preoperative irradiation could be identified by using morphologic changes visualized on US. ${ }^{32}$ A recent study from the Netherlands evaluated 61 patients with advanced-stage head

\begin{tabular}{|c|c|c|c|c|}
\hline & $\begin{array}{l}\text { PPV } \\
(\%)\end{array}$ & $\begin{array}{l}\text { NPV } \\
(\%)\end{array}$ & $\begin{array}{c}\text { Sensitivity } \\
(\%)\end{array}$ & $\begin{array}{c}\text { Specificity } \\
(\%)\end{array}$ \\
\hline US & 11 & 97 & 92 & 28 \\
\hline US-FNA & 33 & 95 & 75 & 74 \\
\hline
\end{tabular}

and neck cancer treated with concurrent chemoradiation showing a sensitivity of $80 \%$ and a specificity of $42 \%$ for postradiotherapy US-guided FNAC, with a yield of $43 \%$ of the salvage neck dissections showing positivity for pathologic evidence of disease. ${ }^{33}$ A study from Singapore found a higher PPV and NPV for US over CT in the evaluation of recurrent nodal adenopathy from nasopharyngeal cancer. ${ }^{34}$

However, legitimate concerns have been raised about performance of US of the neck after RT as an inaccurate and overly formidable technical undertaking. One common belief is that fibrosis in the neck limits examination and makes FNAC samples overly difficult to obtain, and another is that either due to sampling error or idiosyncratically responding nodes at varying stages of posttreatment viability, reliable results are unlikely. ${ }^{35}$ Results obtained from US-guided FNAC are considered highly dependent on operator experience, and in particular, obtaining and analyzing viable FNA from an irradiated tumor mass requires a level of radiologic and pathologic expertise that may not be widely available.

Our study provides some evidence to address these beliefs. Our findings indicate that in experienced hands, US was feasible in all except 1 patient tested. The NPV of a nonsuspicious postradiation US is $97 \%$ (Table 4). Therefore, a posttreatment US may contribute valuable additional reassurance in making a decision to delay neck dissection. In particular, US or serial US may be helpful during the early 3-month window when the interpretation of traditional diagnostic or functional imaging results may be hampered by resolving tumor, edema, and other post-RT effects. ${ }^{36,37}$ The patients sent for US in this study were those who presented with node-positive disease initially and who were selected by clinicians for US referral on the basis of physical findings or imaging characteristics obtained during serial examinations in the immediate postradiation period. Therefore, in the setting of clinical suspicion for residual disease remaining after RT, US is a noninvasive inexpensive form of reassurance with a high NPV.

FNA was also feasible with US guidance. However, $29 \%$ of the aspirates could not provide diagnostic information, perhaps related to radiation of the lymph node. In the presence of positive findings on US though, the NPV was $95 \%$. Thus, either a negative finding on US or a positive finding on US with a negative finding on FNAC was a reliable indicator that avoidance of neck dissection was safe (Table 4).

The PPV and specificity of US were relatively poor (Table 4). The addition of FNAC seemed to contribute minimally in improving the positive yield of a postradiation US but, in some cases, may have been viewed by our clinicians as irrelevant in the face of high clinical suspicion and suspicious US findings. These opinions may lend some credence to the fact that the visible characteristics of US examination in the hands of an experienced operator may be more valuable in practical clinical terms than a needle aspiration after RT, when sampling error and difficulty of interpretation due to treatment effect make the cytologic interpretation more unreliable than usual. 
There are serious barriers to advocating US as a routine surveillance study in patients with head and neck radiation. First, widespread use of US in the head and neck is limited by the specialized training and clinical experience needed to develop a successful US program. Second, proper US interpretation requires an alert and integrated team approach involving the radiation oncologist, sonographer, surgeon, and pathologist; this idealized multimodality decision-making process may not be available at all centers. Results must be evaluated in the context of the timing of the examination, the patient's overall clinical response, and the judgment of the treating clinicians about the degree of nodal response and the likelihood of residual or recurrent disease.

In addition, PET has emerged as a widely available technique for posttreatment evaluation of the head and neck, and many are using PET as the standard examination for the post-RT neck. ${ }^{38}$ Similar to US, the NPV is exceptionally high. ${ }^{39}$ However, PET also has its pitfalls, particularly in producing false-positive results at the primary site ${ }^{40}$ or in the neck when the study is performed too early. PET can sometimes result in the identification of incidental or inflammatory findings, which may trigger unnecessary examination and biopsy. ${ }^{41}$ Our study shows that US may provide corroborating evidence when the PET results are equivocal or may provide a valuable contribution when there is a question of required salvage at a time point when PET is unreliable.

There are some reasons to advocate the continued practice and use of US in postradiation evaluation. Unlike other imaging studies, US can be repeated frequently, and if performed by the same operator, serial studies benefit from greater consistency in observer interpretation. In the future, to develop improved means of distinguishing nodes at high risk of failure, incorporation of biologic and molecular end points will be required, and US offers the unique ability to assess nodes on a pathologic as well as a clinicoradiographic basis. Multiple passes with core needle biopsy may be an alternative technique in appropriately selected patients, which allows improved interpretation. ${ }^{29}$ One study showed that in 43 previously irradiated patients with head and neck cancer who underwent core needle biopsy for suspicion of residual disease, there were 2 cases of sampling error and no falsepositive or false-negative results. ${ }^{42}$ Finally, in an era of escalating health care costs and systemic inefficiency, US remains an inexpensive study that can be performed in the office on short notice. However, qualified experienced head and neck sonographers are uncommon, and if these reasons for preserving the art of neck US are valid, a commitment is needed to train and maintain a pool of expertise for the future.

\section{Conclusions}

US, used in the proper clinical context, offers a noninvasive, anatomically adaptable, and reassuring technique of postradiotherapy neck assessment. A negative finding or a questionable finding on examinations accompanied by a negative finding on a cytologic specimen can reassure the clinician that a neck dissection can be avoided.

\section{References}

1. Baatenburg de Jong RJ, Rongen RJ, Verwoerd CD, et al. Ultrasound-guided fine-needle aspiration biopsy of neck nodes. Arch Otolaryngol Head Neck Surg 1991;117:402-04
2. Hodder SC, Evans RM, Patton DW, et al. Ultrasound and fine needle aspiration cytology in the staging of neck lymph nodes in oral squamous cell carcinoma. Br J Oral Maxillofac Surg 2000;38:430-36

3. Takes RP, Knegt P, Manni JJ, et al. Regional metastasis in head and neck squamous cell carcinoma: revised value of US with US-guided FNAB. Radiology 1996;198:819-23

4. Anand N, Chaudhary N, Mittal MK, et al. Comparison of the efficacy of clinical examination, ultrasound neck and computed tomography in detection and staging of cervical lymph node metastasis in head and neck cancers. Indian J Otolaryngol Head Neck Surg 2006;59:19-23

5. Rottey S, Petrovic M, Bauters W, et al. Evaluation of metastatic lymph nodes in head and neck cancer: a comparative study between palpation, ultrasonography, ultrasound-guided fine needle aspiration cytology and computed tomography. Acta Clin Belg 2006;61:236-41

6. Thakur JS, Sharma ML, Mohan C, et al. Clinicopathological and radiological evaluation of cervical lymph node metastasis in head and neck malignancies. Indian J Otolaryngol Head Neck Surg 2007;59:327-31

7. Ishii J, Amagasa T, Tachibana T, et al. US and CT evaluation of cervical lymph node metastasis from oral cancer. J Craniomaxillofac Surg 1991;19:123-27

8. Amedee RG, Dhurandhar NR. Fine-needle aspiration biopsy. Laryngoscope 2001;111:1551-57

9. Steinkamp HJ, Maurer J, Cornehl M, et al. Recurrent cervical lymphadenopathy: differential diagnosis with color-duplex sonography. Eur Arch Otorhinolaryngol 1994;251:404-09

10. Yonetsu K, Sumi M, Izumi M, et al. Contribution of Doppler sonography blood flow information to the diagnosis of metastatic cervical nodes in patients with head and neck cancer: assessment in relation to anatomic levels of the neck. AJNR Am J Neuroradiol 2001;22:163-69

11. Chikui $\mathrm{T}$, Yuasa $\mathrm{K}$, Tokumori $\mathrm{K}$, et al. Change of sonographic findings on cervical lymph nodes before and after preoperative radiotherapy. Eur Radiol 2004;14:1255-62

12. Ahuja AT, Ying M, Ho SY, et al. Ultrasound of malignant cervical lymph nodes. Cancer Imaging 2008;8:48-56

13. Yusa $H$, Yoshida $H$, Iwasa $S$, et al. Ultrasonographic assessment for response to radiochemotherapy of metastatic cervical lymph nodes in head and neck cancer: usefulness of grey-scale and color Doppler sonography. Ultrasound Med Biol 2000;26:1081-87

14. McHam SA, Adelstein DJ, Rybicki LA, et al. Who merits a neck dissection after definitive chemoradiotherapy for N2-N3 squamous cell head and neck cancer? Head Neck 2003;25:791-98

15. Parsons JT, Million RR, Cassisi NJ. Carcinoma of the base of the tongue: results of radical irradiation with surgery reserved for irradiation failure. Laryngoscope 1982;92:689-96

16. Roy S, Tibesar RJ, Daly K, et al. Role of planned neck dissection for advanced metastatic disease in tongue base or tonsil squamous cell carcinoma treated with radiotherapy. Head Neck 2002;24:474-81

17. Barkley HT Jr, Fletcher GH, Jesse RH, et al. Management of cervical lymph node metastases in squamous cell carcinoma of the tonsillar fossa, base of tongue, supraglottic larynx, and hypopharynx. Am J Surg 1972;124:462-67

18. Mendenhall WM, Million RR, Cassisi NJ. Squamous cell carcinoma of the head and neck treated with radiation therapy: the role of neck dissection for clinically positive neck nodes. Int J Radiat Oncol Biol Phys 1986;12:733-40

19. Narayan $\mathrm{K}$, Crane $\mathrm{CH}$, Kleid S, et al. Planned neck dissection as an adjunct to the management of patients with advanced neck disease treated with definitive radiotherapy: for some or for all? Head Neck 1999;21:606-13

20. Robbins KT, Wong FS, Kumar P, et al. Efficacy of targeted chemoradiation and planned selective neck dissection to control bulky nodal disease in advanced head and neck cancer. Arch Otolaryngol Head Neck Surg 1999;125:670-75

21. Weisman RA, Robbins KT. Management of the neck in patients with head and neck cancer treated by concurrent chemotherapy and radiation. Otolaryngol Clin North Am 1998;31:773-84

22. Corry J, Peters L, Fisher R, et al. N2-N3 neck nodal control without planned neck dissection for clinical/radiologic complete responders: results of Trans Tasman Radiation Oncology Group Study 98.02. Head Neck 2008;30:737-42

23. Liauw SL, Mancuso AA, Amdur RJ, et al. Postradiotherapy neck dissection for lymph node-positive head and neck cancer: the use of computed tomography to manage the neck. J Clin Oncol 2006;24:1421-27

24. Moeller BJ, Rana V, Cannon BA, et al. Prospective risk-adjusted [18F]fluorodeoxyglucose positron emission tomography and computed tomography assessment of radiation response in head and neck cancer. J Clin Oncol 2009;27: 2509-15. Epub 2009 Mar 30

25. Ahuja A, Ying M, King W, et al. A practical approach to ultrasound of cervical lymph nodes. J Laryngol Otol 1997;111:245-56

26. Akbar NA, Bodenner DL, Kim LT, et al. Considerations in incorporating office-based ultrasound of the head and neck. Otolaryngol Head Neck Surg 2006;135:884-88

27. Castelijns JA, van den Brekel MW. Imaging of lymphadenopathy in the neck. Eur Radiol 2002;12:727-38

28. Howlett DC, Harper B, Quante M, et al. Diagnostic adequacy and accuracy of fine needle aspiration cytology in neck lump assessment: results from a regional cancer network over a one year period. J Laryngol Otol 2007;121:571-79 
29. Howlett DC, Mercer J, Williams MD. Same day diagnosis of neck lumps using ultrasound-guided fine-needle core biopsy. $\mathrm{Br}$ J Oral Maxillofac Surg 2008;46:64-65

30. van den Brekel MW, Castelijns JA, Reitsma LC, et al. Outcome of observing the N0 neck using ultrasonographic-guided cytology for follow-up. Arch Otolaryngol Head Neck Surg 1999;125:153-56

31. Westhofen M. Ultrasound B-scans in the follow-up of head and neck tumors. Head Neck Surg 1987;9:272-78

32. Hessling $\mathrm{KH}$, Schmelzeisen $\mathrm{R}$, Reimer $\mathrm{P}$, et al. Use of sonography in the follow-up of preoperatively irradiated efferent lymphatics of the neck in oropharyngeal tumours. J Craniomaxillofac Surg 1991;19:128-30

33. van der Putten L, van den Broek GB, de Bree R, et al. Effectiveness of salvage selective and modified radical neck dissection for regional pathologic lymphadenopathy after chemoradiation. Head Neck 2009;31:593-603

34. Toh ST, Yuen HW, Goh YH, et al. Evaluation of recurrent nodal disease after definitive radiation therapy for nasopharyngeal carcinoma: diagnostic value of fine-needle aspiration cytology and CT scan. Head Neck 2007;29:370-77

35. Ahuja AT, Evans RM. Practical Head and Neck Ultrasound. London: Greenwich Medical Media Limited; 2000

36. Ahuja AT, Ho SS, Leung SF, et al. Metastatic adenopathy from nasopharyngeal carcinoma: successful response to radiation therapy assessed by color duplex sonography. AJNR Am J Neuroradiol 1999;20:151-56

37. Yuasa K, Kawazu T, Kunitake N, et al. Sonography for the detection of cervical lymph node metastases among patients with tongue cancer: criteria for early detection and assessment of follow-up examination intervals. AJNR Am J Neuroradiol 2000;21:1127-32

38. Pellitteri PK, Ferlito A, Rinaldo A, et al. Planned neck dissection following chemoradiotherapy for advanced head and neck cancer: is it necessary for all? Head Neck 2006;28:166-75

39. Yao M, Luo P, Hoffman HT, et al. Pathology and FDG PET correlation of residual lymph nodes in head and neck cancer after radiation treatment. Am J Clin Oncol 2007;30:264-70

40. Yao M, Smith RB, Hoffman HT, et al. Clinical significance of postradiotherapy [18F]-fluorodeoxyglucose positron emission tomography imaging in management of head-and-neck cancer: a long-term outcome report. Int J Radiat Oncol Biol Phys 2009;74:9-14

41. Ryan WR, Fee WE Jr, Le QT, et al. Positron-emission tomography for surveillance of head and neck cancer. Laryngoscope 2005;115:645-50

42. Pfeiffer J, Kayser L, Ridder GJ. Minimal-invasive core needle biopsy of head and neck malignancies: clinical evaluation for radiation oncology. Radiother Oncol 2009;90:202-07 